\title{
A Nomogram to Predict Symptomatic Intracranial Hemorrhage After Intravenous Thrombolysis in Chinese Patients
}

\author{
Hongquan Guo, (D) ${ }^{1, *}$ \\ Wei Xu, ${ }^{1,2, *}$ \\ Xiaohao Zhang, ${ }^{3, *}$ \\ Shuai Zhang, ${ }^{4}$ Zheng Dai, ${ }^{5}$ \\ Shun $\mathrm{Li}^{6}{ }^{6} \mathrm{Yi}_{\mathrm{X}} \mathrm{Xie}{ }^{3}$ \\ Yingle Li, (D) ${ }^{\prime}$ \\ Jianzhong Xue, ${ }^{7}$ \\ Xinfeng Liu'
}

'Department of Neurology, Jinling Hospital, The First School of Clinical Medicine, Southern Medical University, Nanjing, Jiangsu, 210002 , People's Republic of China; 'Department of Neurology, Affiliated Changsha Central Hospital, University of South China, Changsha, Hunan, 4I0000, People's Republic of China; ${ }^{3}$ Department of Neurology, jinling Hospital, Medical School of Nanjing University, Nanjing, Jiangsu, 210002 , People's Republic of China; ${ }^{4}$ Department of Neurology, The Affiliated Hospital of Yangzhou University, Yangzhou University, Yangzhou, Jiangsu, 22500 I, People's Republic of China; ${ }^{5}$ Department of Neurology, The Affiliated Wuxi People's Hospital of Nanjing Medical University, Wuxi, Jiangsu, 21 4023, People's Republic of China; ${ }^{6}$ Department of Cerebrovascular Disease Treatment Center, Nanjing Brain Hospital Affiliated to Nanjing Medical University, Nanjing, Jiangsu, 210002 , People's Republic of China; ${ }^{7}$ Department of Neurology, Changshu No.2 People's Hospital, Changshu, Jiangsu, 215500, People's Republic of China

*These authors contributed equally to this work

Correspondence: Xinfeng Liu

Department of Neurology, Jinling Hospital, The First School of Clinical Medicine, Southern Medical University, No. 305 East Zhongshan Road, Nanjing, 210002, Jiangsu

Province, People's Republic of China

$\mathrm{Tel}+86258480186 \mathrm{I}$

Fax +86 2584805I69

Email xfliu2@vip.163.com

Jianzhong Xue

Department of Neurology, Changshu No.2

People's Hospital, No. 18 Taishan Road,

Changshu, Jiangsu Province, People's

Republic of China

Tel +86 I3506247572

Email xjz2021@sina.com
Background and Aims: A reliable predictive score system to identify the risk of symptomatic intracranial hemorrhage ( $\mathrm{sICH}$ ) after intravenous thrombolysis (IVT) in acute ischemic stroke patients is of great essence. We aimed to develop a nomogram for predicting the risk of sICH after IVT in Chinese patients.

Methods: We recruited acute ischemic stroke patients who were treated with IVT from five advanced stroke centers in China from April 2014 to November 2020. sICH was diagnosed according to the European Cooperative Acute Stroke Study II (ECASS-II) definition. Multivariable logistic regression was performed to construct the best-fit nomogram. The discrimination and calibration of the nomogram were evaluated by the area under the receiver operating characteristic curve (AUC-ROC) and calibration plot.

Results: A total of 1200 patients were enrolled, of whom 66 (5.5\%) developed sICH. In the multivariate logistic regression model, atrial fibrillation (odds ratio [OR] 3.25; 95\% confidence interval [CI], 1.89-5.60; $P<0.001)$, baseline glucose level (OR, 1.13; 95\% CI, 1.07 $-1.20 ; P<0.001$ ), neutrophil to lymphocyte ratio (OR, $1.05 ; 95 \% \mathrm{CI}, 1.01-1.09 ; P=0.024)$ and baseline National Institute of Health Stroke Scale (NIHSS) (OR, 1.07; 95\% CI, 1.04 $-1.10 ; P<0.001)$ were independent predictors for sICH and were used to generate the nomogram. The nomogram demonstrated good discrimination as the AUC-ROC value was 0.788 (95\% CI, 0.737-0.840). The calibration plot revealed good calibration.

Conclusion: The nomogram consisted of atrial fibrillation, baseline glucose level, neutrophil to lymphocyte ratio, and NIHSS score may predict the risk of sICH in Chinese acute ischemic stroke patients treated with IVT.

Keywords: stroke, intravenous thrombolysis, symptomatic intracranial hemorrhage, nomogram, predict

\section{Introduction}

Although endovascular treatment has been a promising treatment for acute ischemic stroke in recent years, intravenous thrombolysis (IVT) now still be a preferred and effective treatment for patients within the time window of 4.5 hours. ${ }^{1}$ Symptomatic intracranial hemorrhage (sICH) is a rare but dangerous complication for IVT and limits its widespread use. ${ }^{2}$ Due to different study populations and sICH definitions, the $\mathrm{sICH}$ rate ranges from $2.0 \%$ to $7.0 \%{ }^{3}$ Compared with other race/ethnic groups, the Asian population might take a higher risk for sICH. ${ }^{4}$

Since sICH is significantly associated with poor outcomes, a reliable prognostic model to identify patients with increased risk of sICH after IVT is essential. There 
have been several prognostic score systems to predict the risk of sICH after IVT. ${ }^{5-11}$ However, these score systems usually convert continuous variables such as age, blood glucose, NIHSS score to categorical variables, which might lead to loss of information because these factors are strong predictors of sICH after IVT.

Nomogram is a graphical statistical tool that uses a continuous score to calculate the probability of a clinical event for an individual patient and has been widely used in modern individualized medical decisionmaking. ${ }^{12-15}$ The STARTING-SICH nomogram was designed for prediction of sICH in stroke patients treated with IVT. ${ }^{16}$ However, this nomogram was derived from European-American ancestry. Therefore, it is necessary to develop a novel nomogram in the Asian population. Based on a multicenter database derived from 5 stroke centers, the present study aimed to develop a nomogram to predict the probability of sICH after IVT in Chinese stroke patients.

\section{Methods}

\section{Study Population}

The study was a retrospective cohort analysis based on prospectively collected data from five advanced stroke centers in China between April 2014 and November 2020 (including Jinling Hospital, Changsha Central Hospital, Affiliated Hospital of Yangzhou University, Wuxi People's Hospital, and Nanjing Brain Hospital). Inclusion criteria: 1) age $\geq 18$ years; 2) diagnosed with acute ischemic stroke; 3) treated with IVT. Patients treated with endovascular treatment after IVT, patients with severe inflammatory diseases, and those with incomplete clinical data were excluded from the analysis. This study was approved by the ethics committee of Jinling Hospital and each participating center. Due to its retrospective nature; patient consent was waived. All procedures performed in studies were under the 1964 Helsinki declaration and its later amendments or comparable ethical standards. Patient data was confidentiality maintained in Jingling Hospital and each participating center.

\section{Baseline Data Collection}

Demographic characteristics, medical history, clinical data were obtained at admission. Stroke severity was evaluated by the National Institutes of Health Stroke Scale (NIHSS) score. Laboratory data including baseline blood glucose, neutrophil-to-lymphocyte ratio (NLR), platelet, total cholesterol, triglyceride, low-density lipoprotein and highdensity lipoprotein were also recorded. NLR value was computed as neutrophil counts/lymphocyte counts.

\section{Definition of sICH}

All patients enrolled in the study underwent a CT scan within 24 hours after IVT and performed another CT whenever neurological deterioration appears. The $\mathrm{sICH}$ was diagnosed based on the European Cooperative Acute Stroke Study II (ECASS-II) definition: any type of intracerebral hemorrhage on post-thrombolysis imaging and increase of $\geq 4$ NIHSS points from baseline, or the lowest score within 7 days, or leading to death. ${ }^{17}$ All image data were independently reviewed by two neurologists to assess the presence of sICH. In case of disagreement, final results were sought by consensus discussed with a third neurologist.

\section{Statistical Analysis}

Descriptive analysis was conducted as follows: continuous variables were presented as medians with interquartile ranges (IQRs) or mean \pm standard deviation (SD); categorical variables were described as numbers with percentages. Differences between the groups with and without sICH were explored using the Mann-Whitney $U$-test or $t$-student test for continuous variables as appropriate. Differences between the two groups for categorical variables were analyzed by Fisher exact test or $\chi^{2}$ test, when appropriate. To construct the nomogram, we implemented a multivariate logistic regression analysis using the forward stepwise method to determine independent factors of sICH, all variables with $P$ value $<0.1$ in the univariate analysis were included. Variables with $P$ value $<0.05$ in the multivariate logistic regression were entered to generate the predictive model. Regression coefficient and odds ratios (OR) with $95 \%$ confidence intervals (CI) for each variable included in the model were calculated. The regression coefficient of each variable in the model was used to compute corresponding points in the scale and, eventually, obtaining the scoring system. The discriminative ability of the novel nomogram was assessed by calculating the area under the receiver-operating characteristic curve (AUC-ROC). Calibration of the predictive model that described the concordance between the observed and predicted probability based on the nomogram was tested using a calibration plot with bootstraps of 1000 resamples. All statistical analyses were performed using statistical 
software SPSS version 22.0 (IBM, New York, NY) and $\mathrm{R}$ version 3.0 (R Foundation, Vienna, Austria).

\section{Results}

The flow chart of patient inclusion and exclusion is presented in Figure 1. There were 1200 acute ischemic stroke patients treated with IVT finally included in the present study to construct the nomogram. The clinical characteristics of patients are presented in Table 1 . The mean age of the total group was 66.9 years, and $63.0 \%$ were male. According to the ECASS II definition, 66 patients (5.5\%) were classified as sICH after IVT. Compared with patients without sICH, sICH patients were older (mean, 72.2 versus 66.6 years; $P<0.001$ ), more prone to have a history of atrial fibrillation $(47.0 \%$ versus $16.6 \% ; P<0.001)$, and had higher baseline blood glucose (mean, 9.8 versus 7.9 $\mathrm{mmol} / \mathrm{L}, P<0.001$ ), NLR (median, 3.50 versus 2.74; $P=$ 0.002 ), and baseline NIHSS (median, 15 versus 7; $P<$ 0.001 ), and had lower platelet (mean, 178.0 versus 198.4 $10^{9} / \mathrm{L} ; P=0.007$ ) (Table 1 ).

The results of the multivariate logistic regression model are shown in Table 2. Using the forward stepwise method, the following 4 variables were independent risk factors of sICH: atrial fibrillation (OR 3.25; 95\% CI, 1.89 -5.60; $P<0.001$ ), baseline glucose level (OR, 1.13; 95\% CI, $1.07-1.20 ; P<0.001)$, NLR (OR, $1.05 ; 95 \%$ CI, 1.01 $-1.09 ; P=0.024)$ and baseline NIHSS (OR, 1.07; 95\% CI, $1.04-1.10 ; P<0.001$ ). Using the predictors (atrial fibrillation, baseline glucose level, NLR, baseline NIHSS) in the multivariable model, we constructed the nomogram to predict sICH risk. The nomogram was created by assigning a graphic preliminary score to each of the four predictors with a point ranging from 0-100, and then summed up all the points of each predictor to

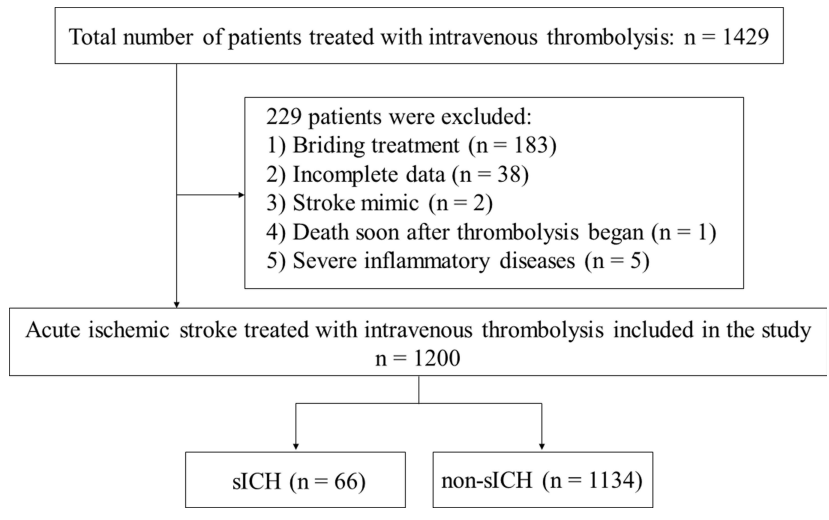

Figure I Flow chart of patients included in the study. Abbreviation: slCH, symptomatic intracranial hemorrhage. obtain the total point, eventually converted into a percentage representing an individual probability of sICH after IVT. The detailed nomogram is shown in Figure 2. For example, a patient with atrial fibrillation, blood glucose at $20 \mathrm{mmol} / \mathrm{L}$, baseline NIHSS at 20 points, NLR at 10 would have a total of 125 points ( 27 points for atrial fibrillation, 57 points for blood glucose, 30 points for baseline NIHSS, and 11 points for NLR). The predicted sICH risk after IVT was approximately $52.0 \%$ for this patient.

We calculated the AUC-ROC value to investigate the discriminative ability of the nomogram, which was 0.788 (95\% CI, 0.737-0.840) (Figure 3). The calibration plot revealed good predictive accuracy between the actual probability and predicted probability (Figure 4).

\section{Discussion}

In this study, we found that the presence of atrial fibrillation, baseline glucose level, NLR, and NIHSS score were independent predictors for sICH after IVT. Based on the four independent factors, we constructed a nomogram to individually predict the probability of sICH for acute ischemic stroke patients treated with IVT in the Chinese population.

We found that 66 patients $(5.5 \%)$ experienced $\mathrm{sICH}$ after IVT in the present study. Raymond C.S. Seet reviewed the incidence of sICH following IVT for acute ischemic stroke, including randomized controlled trials, registry studies, and cohort studies, and found the mean sICH rates for these studies were $7.5 \%, 3.5 \%$, and $5.9 \%$, respectively, and the mean overall incidence of sICH was $5.6 \%{ }^{3}$ The difference in rates of sICH among studies might be owing to the different definitions of sICH and study populations. Prior studies show that the ECASS-II criteria may have the highest interrater agreement and are strongly correlated with poor outcome at 3 months. ${ }^{2,18}$ The sICH incidence in our study was a little higher than that reported in the STARTING-SICH cohort (3.6\%), which was conducted in the European-American ancestry ${ }^{16}$ because the Asian population may have a higher risk of sICH than other race/ethnic groups. ${ }^{4}$

Consistent with prior studies, our study found that atrial fibrillation was associated with sICH after IVT. Hu et al carried out a meta-analysis about the safety of IVT for acute ischemic stroke with atrial fibrillation and found that the incidence of sICH in atrial fibrillation patients was higher than that in non-atrial fibrillation patients $(6.4 \%$ vs $4.1 \% ; P<$ 0.001). ${ }^{19}$ The following points might explain the reason that 
Table I Comparison of Demographics and Clinical Data in Patients with or without sICH

\begin{tabular}{|c|c|c|c|}
\hline Variable & $\begin{array}{c}\text { with sICH } \\
n=66\end{array}$ & $\begin{array}{c}\text { without sICH } \\
n=1 / 34\end{array}$ & $P$ value \\
\hline \multicolumn{4}{|l|}{ Demographics } \\
\hline Age, years & $72.2 \pm 11.8$ & $66.6 \pm 12.5$ & $<0.001$ \\
\hline Male, n (\%) & $37(56.1)$ & $719(63.4)$ & 0.23 \\
\hline Weight, kg & $63.4 \pm 13.7$ & $64.5 \pm 11.7$ & 0.224 \\
\hline \multicolumn{4}{|l|}{ Medical history, n (\%) } \\
\hline Hypertension & $47(7 \mid .2)$ & $796(70.2)$ & 0.86 \\
\hline Diabetes mellitus & $14(2 \mid .2)$ & $275(24.3)$ & 0.575 \\
\hline Hyperlipidemia & $\mathrm{I}(\mathrm{I} .5)$ & $57(5.0)$ & 0.196 \\
\hline Atrial fibrillation & $31(47.0)$ & $188(16.6)$ & $<0.001$ \\
\hline Smoking & $21(31.8)$ & $381(33.6)$ & 0.766 \\
\hline Coronary heart disease & $16(24.2)$ & $208(18.3)$ & 0.232 \\
\hline Previous stroke & $13(19.7)$ & $224(19.8)$ & 0.991 \\
\hline \multicolumn{4}{|l|}{ Clinical data } \\
\hline Systolic blood pressure, $\mathrm{mmHg}$ & $161.4 \pm 29.8$ & $155.3 \pm 26.4$ & 0.063 \\
\hline Diastolic blood pressure, $\mathrm{mmHg}$ & $91.4 \pm 17.3$ & $87.1 \pm 15.3$ & 0.056 \\
\hline Time from onset to treatment, $\mathrm{min}$ & $174(128,219)$ & $165(126,209)$ & 0.295 \\
\hline Baseline NIHSS, score & $15(I I, 21)$ & $7(4,13)$ & $<0.001$ \\
\hline \multicolumn{4}{|l|}{ Laboratory data } \\
\hline Platelet, $10^{9} / \mathrm{L}$ & $178.0 \pm 51.4$ & $198.4 \pm 63.5$ & 0.007 \\
\hline Total cholesterol, $\mathrm{mmol} / \mathrm{L}$ & $3.4 \pm 1.7$ & $3.5 \pm 1.8$ & 0.853 \\
\hline Triglyceride, $\mathrm{mmol} / \mathrm{L}$ & $\mathrm{I} .24(0.9 \mathrm{I}, 3.44)$ & $1.8(1.12,4.04)$ & 0.105 \\
\hline Low density lipoprotein, $\mathrm{mmol} / \mathrm{L}$ & $1.94(0.77,2.73)$ & $2.1(0.62,2.84)$ & 0.927 \\
\hline High-density lipoprotein, mmol/L & $1.6 \pm 0.8$ & $1.5 \pm 0.8$ & 0.153 \\
\hline Baseline blood glucose, $\mathrm{mmol} / \mathrm{L}$ & $9.8 \pm 3.2$ & $7.9 \pm 3.4$ & $<0.001$ \\
\hline NLR & $3.50(2.18,7.43)$ & $2.74(1.80,4.46)$ & 0.002 \\
\hline
\end{tabular}

Abbreviations: sICH, symptomatic intracranial hemorrhage; NIHSS, National Institute of Health Stroke Scale; NLR, neutrophil-to-lymphocyte ratio.

atrial fibrillation patients have a higher risk of sICH after IVT: First, atrial fibrillation patients are more likely to have old and organized thrombi, which are often insensitive to IVT, leading to the time extension of recanalization, and increase the risk of bleeding. ${ }^{20}$ Second, patients with atrial fibrillation usually have poor collateral circulation, severe intracranial hypoperfusion, and large infarct sizes, thus increase the reperfusion hemorrhage risk. $^{21}$ Third, a subgroup of atrial fibrillation patients take anticoagulant drugs before IVT, and it may also increase the bleeding risk. Higher baseline glucose level is another predicted factor in our nomogram model. Hyperglycemia can result in increased matrix metalloproteinase- 9 and a series of oxidative stress reactions, which may disrupt the integrity of the blood brain barrier and increase the permeability of the blood brain barrier, leading to the development of sICH. ${ }^{22}$ Baseline NIHSS score, which reflects the severity of the stroke, has been confirmed as the risk factor for sICH following
IVT. ${ }^{9,23,24}$ Our nomogram model indicated that patients with higher baseline NIHSS score might have an increased risk of sICH.

Our study also revealed that NLR was an independent risk factor for sICH. The inflammatory response mediated by the migration of inflammatory cells and the activation of extracellular matrix kinase (mainly matrix metalloproteinases), which leading to the disruption of blood brain barrier integrity has been proven to play a vital role in hemorrhagic transformation. ${ }^{25,26}$ The neutrophils can increase the permeability of the blood-brain barrier by release related cytokines, chemokines, adhesion molecules, and different proteases, while the activation of lymphocytes may reduce the destruction of the blood-brain barrier. As the balance between neutrophils and lymphocytes, NLR is considered the biomarker for systemic inflammation. Maestrini et al reported that NLR was the best predictor for SICH after IVT, and an NLR $\geq 4.8$ before 
Table 2 Multivariate Regression Analysis for sICH

\begin{tabular}{|c|c|c|c|c|c|}
\hline \multirow[t]{2}{*}{ Variable } & \multicolumn{2}{|c|}{ Univariate Regression Model } & \multicolumn{3}{|c|}{ Multivariate Regression Model } \\
\hline & OR (95\% Cl) & $P$ value & Regression Coefficient & OR $(95 \% \mathrm{Cl})$ & $P$ value \\
\hline Age, years & $1.04(1.02-1.06)$ & 0.001 & & & \\
\hline Atrial fibrillation & $4.46(2.68-7.4 I)$ & $<0.001$ & 1.18 & $3.25(1.89-5.60)$ & $<0.001$ \\
\hline Systolic blood pressure, $\mathrm{mmHg}$ & $1.01(0.99-1.02)$ & 0.073 & & & \\
\hline Diastolic blood pressure, $\mathrm{mmHg}$ & $1.02(1.00-1.03)$ & 0.027 & & & \\
\hline Baseline NIHSS, score & $1.08(1.05-1.11)$ & $<0.001$ & 0.064 & $1.07(1.04-1.10)$ & $<0.001$ \\
\hline Platelet, $10^{9} / \mathrm{L}$ & $0.99(0.99-1.00)$ & 0.01 & & & \\
\hline Baseline glucose (per I mmol/l increase) & $1.12(1.06-1.19)$ & $<0.001$ & 0.123 & $1.13(1.07-1.20)$ & $<0.001$ \\
\hline NLR & $1.06(1.03-1.10)$ & 0.001 & 0.047 & $1.05(1.01-1.09)$ & 0.024 \\
\hline
\end{tabular}

Abbreviations: $\mathrm{Cl}$, confidence interval; OR, odd ratio; NIHSS, National Institute of Health Stroke Scale; NLR, neutrophil-to-lymphocyte ratio; sICH, symptomatic intracranial hemorrhage.

thrombolysis showed a 3.7 -fold increased risk for sICH. ${ }^{27}$ A clinical study from Guo et al reported that the dynamic change of NLR was associated with sICH after IVT in acute ischemic stroke patients. ${ }^{28}$ To our knowledge, our study is the first predictive score system that includes NLR as a predictor to predict the probability of sICH after IVT.

Several predictive scoring systems have been published for predicting sICH after IVT in acute ischemic stroke patients $^{5-11}$ (Table 3). However, these scores often converted the discrete/continuous variables into a dichotomous or multi-categorical variable, thus might lead to the loss of information and decrease the predictive accuracy. Nomogram is a graphical calculation instrument that can assign an accurate numerical probability of a clinical event for an individual patient, which bypasses the categorization of continuous variables into $\geq 2$ different groups. ${ }^{12}$ The STARTING-SICH predicted model composed of 10 clinical variables was the first nomogram model to predict the risk

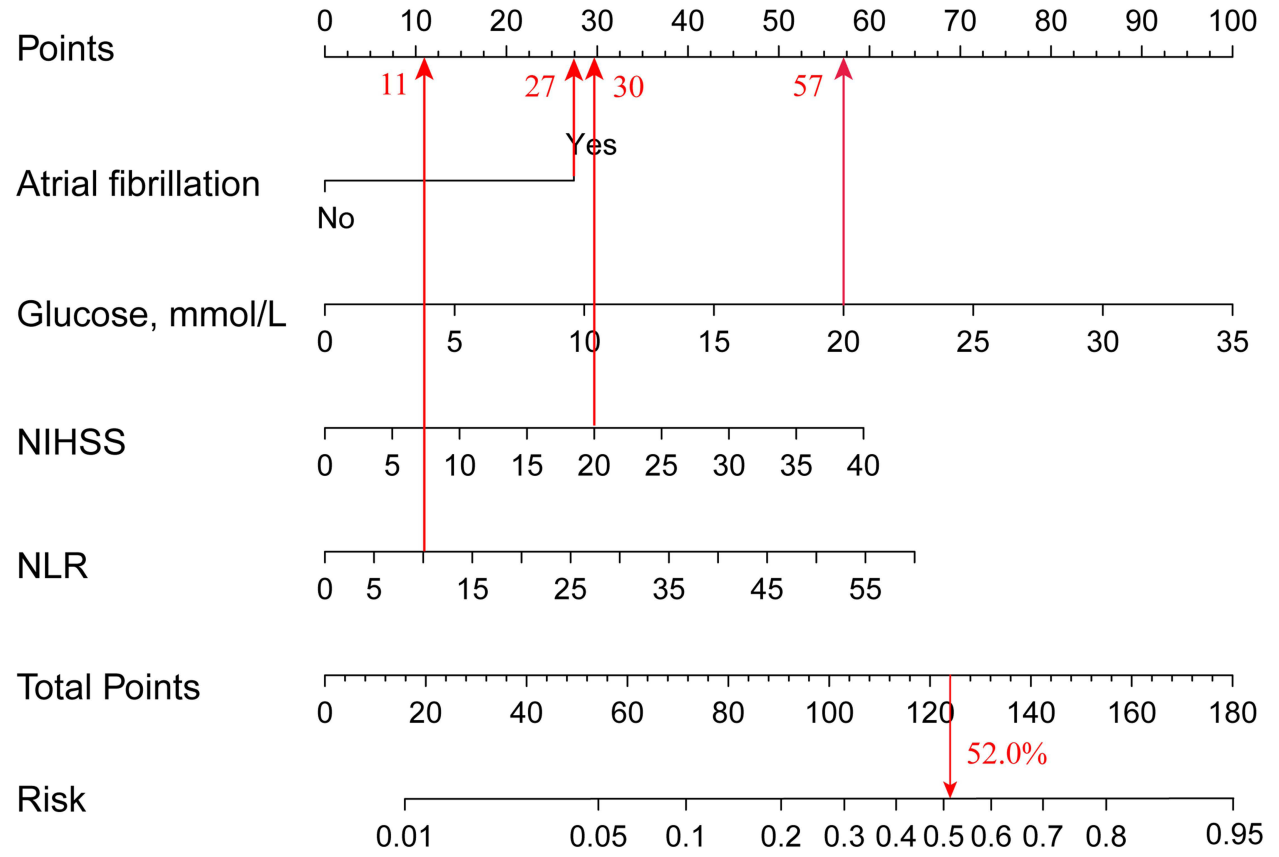

Figure 2 The nomogram to predict the probability of $\mathrm{sICH}$ in stroke patients treated with intravenous thrombolysis.

Abbreviations: sICH, symptomatic intracranial hemorrhage; NIHSS, National Institute of Health Stroke Scale; NLR, neutrophil-to-lymphocyte ratio. 


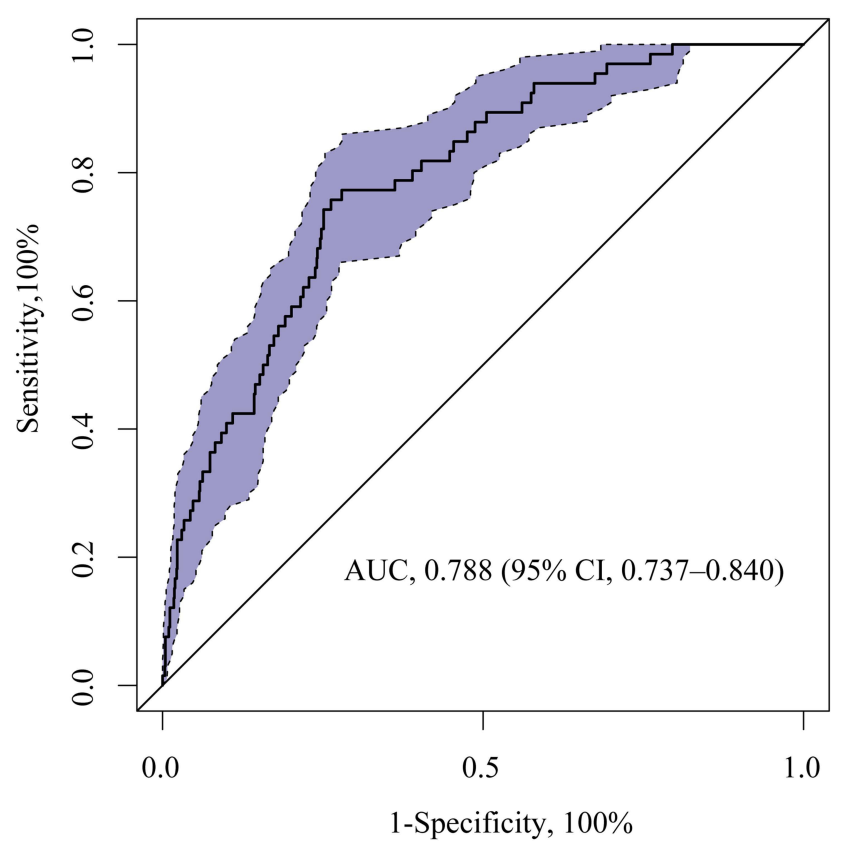

Figure $3 \mathrm{ROC}$ curve of the nomogram for predicting $\mathrm{sICH}$ in stroke patients treated with intravenous thrombolysis.

Abbreviations: AUC, area under the ROC curve; ROC, receiver operating characteristic; sICH, symptomatic intracranial hemorrhage.

probability of sICH after IVT, and it was conducted in the European-American ancestry. ${ }^{16}$ Considering the differences in sICH risk between European-American and Asian populations, it is necessary to construct a nomogram to predict the risk of sICH in Asians. As far as we know, our predictive model is the first nomogram to predict sICH risk in Asian populations. There were a good discriminative and a calibration ability for our nomogram model. With this nomogram, clinicians can easily and quickly discern patients who are at increased risk of developing $\mathrm{sICH}$ that require thrombolysis, take intensive monitoring for an individual patient, and also help guide the expectations of patients and their families.

The strengths of our study are as follows: the data are derived from multiple stroke centers in China, the sample size is relatively large, and the prognostic factors included in the nomogram can be easily and quickly achieved at admission. Nevertheless, there are some limitations to our study. First, this is a retrospective analysis of multicenter data, the accuracy of the predictive model may be influenced by missing data. Second, several neuroradiologic predictors, such as early infarction signs and hyperdense middle cerebral artery signs were not available in the study. Our nomogram might be suitable for those who cannot accurately interpret the image of early infarction signs. Third, although we tried to include study parameters as many as possible, some major confounders which may influence sICH were not available, such as previous anticoagulant use, previous antiplatelet agents use, blood coagulation status on admission, blood pressure variability, and cerebral microbleeds. Finally, further external validations in other different cohorts are still warranted.

\section{Conclusions}

In conclusion, we developed a novel nomogram predictive model, which is consisted of atrial fibrillation, baseline glucose level, NIHSS score and NLR to predict the risk probability of sICH after IVT treatment in Chinese

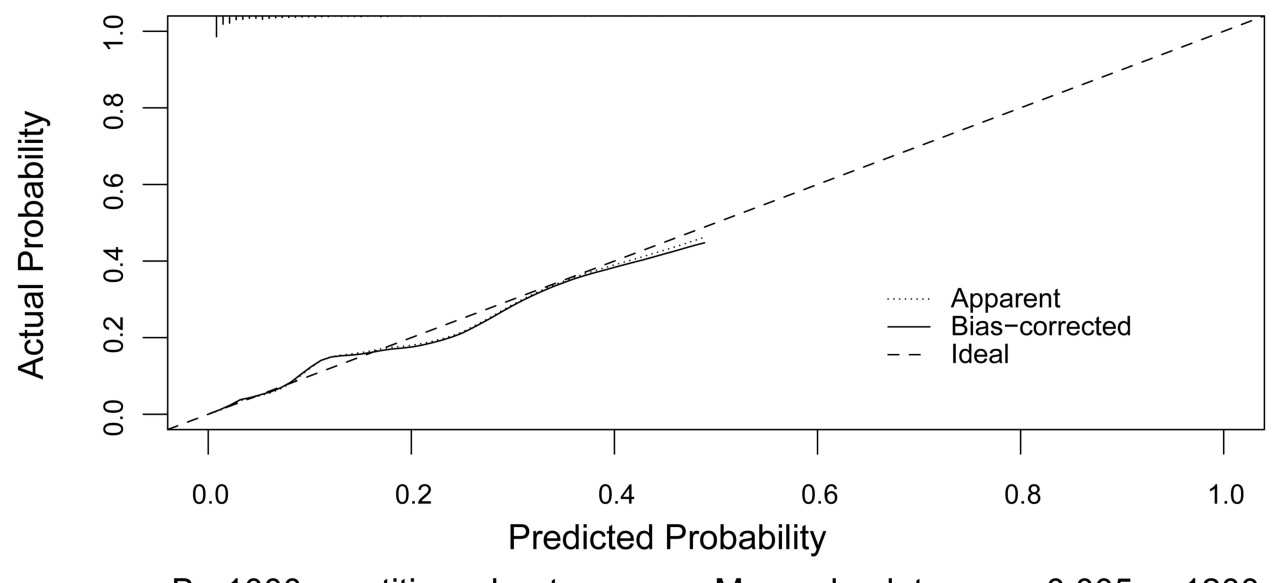

Figure 4 Calibration plot of the nomogram model. Dotted line is the performance of the nomogram, while the solid line corrects for any bias in the nomogram. Dashed line is the reference line where a nomogram would lie. 
Table 3 Description of the Representative sICH Predictive Model

\begin{tabular}{|l|l|c|}
\hline $\begin{array}{l}\text { Predictive } \\
\text { Model }\end{array}$ & Variables in the Model & $\begin{array}{c}\text { Definition of } \\
\text { sICH }\end{array}$ \\
\hline GRASPS & Age, sex, ethnicity, NIHSS, blood glucose, systolic blood pressure & NINDS \\
\hline HAT & Diabetes mellitus /blood glucose, NIHSS, hypodensity on CT (MCA territory) & ECASS-II \\
\hline SEDAN & Age, NIHSS, blood glucose, early infarct signs, hyperdense cerebral artery sign & SITS-MOST \\
\hline SITS-SICH & $\begin{array}{l}\text { Age, body weight, hypertension, NIHSS, blood glucose, systolic blood pressure, onset to treatment time, } \\
\text { aspirin alone, aspirin plus clopidogrel }\end{array}$ & NINDS \\
\hline THRIVE & Age, NIHSS, hypertension, diabetes mellitus, and atrial fibrillation & NINDS \\
\hline SPAN-I00 & Age, NIHSS & ECASS-II \\
\hline $\begin{array}{l}\text { STARTING- } \\
\text { SICH }\end{array}$ & $\begin{array}{l}\text { Age, aspirin alone, aspirin plus clopidogrel, oral anticoagulant with INR } \leq \text { I.7, pre-stroke modified Rankin Scale } \\
\text { sign, current infarction sign }\end{array}$ & \\
\hline
\end{tabular}

Abbreviations: sICH, symptomatic intracranial hemorrhage; NIHSS, National Institute of Health Stroke Scale; SITS, Safe Implementation of Thrombolysis in Stroke; SITSMOST, Safe Implementation of Thrombolysis in Stroke-Monitoring Study; GRASPS, Glucose Race Age Sex Pressure Stroke Severity; HAT, hemorrhage after thrombolysis; SEDAN, blood sugar, early infarct signs, hyperdense cerebral artery sign, age, NIH Stroke Scale; THRIVE, totaled health risks in vascular events; SPAN-I00, stroke prognostication using age and NIH stroke scale-100; STARTING-SICH, systolic blood pressure, age, onset-to-treatment time for thrombolysis, NIHSS score, glucose, aspirin alone, aspirin plus clopidogrel, anticoagulant with INR $\leq 1.7$, current infarction sign, hyperdense artery sign; NINDS, National Institute of Neurological Disorders and Stroke; ECASS-II, European Cooperative Acute Stroke Study II.

acute ischemic stroke patients. Further external validation is warranted to validate the efficacy of the nomogram in different populations.

\section{Data Sharing Statement}

The data that support the findings of this study are available on request from the corresponding author (Xinfeng Liu).

\section{Acknowledgments}

We express our gratitude to all the researchers and patients who participated in this study.

\section{Funding}

This study was supported in part by the National Natural Science Foundation of China (NO. U20A20357, 81870947), the National Key R\&D project (NO. 2017YFC1307901) and Key R\&D Program of Jiangsu Province (Clinical Frontier Technology Project) (NO. BE2020700).

\section{Disclosure}

All the authors declare that there are no conflicts of interest.

\section{References}

1. Powers WJ, Rabinstein AA, Ackerson T, et al. 2018 Guidelines for the early management of patients with acute ischemic stroke: a guideline for healthcare professionals from the American heart association/ American stroke association. Stroke. 2018;49(3):e46-e110. doi:10.1161/STR.0000000000000158

2. Strbian D, Sairanen T, Meretoja A, et al. Patient outcomes from symptomatic intracerebral hemorrhage after stroke thrombolysis. Neurology. 2011;77(4):341-348. doi:10.1212/ WNL.0b013e3182267b8c

3. Seet RC, Rabinstein AA. Symptomatic intracranial hemorrhage following intravenous thrombolysis for acute ischemic stroke: a critical review of case definitions. Cerebrovasc Dis. 2012;34(2):106-114. doi:10.1159/000339675

4. Mehta RH, Cox M, Smith EE, et al. Race/Ethnic differences in the risk of hemorrhagic complications among patients with ischemic stroke receiving thrombolytic therapy. Stroke. 2014;45(8):2263-2269. doi:10.1161/STROKEAHA.114.005019

5. Menon BK, Saver JL, Prabhakaran S, et al. Risk score for intracranial hemorrhage in patients with acute ischemic stroke treated with intravenous tissue-type plasminogen activator. Stroke. 2012;43(9):2293-2299. doi:10.1161/STROKEAHA.112.660415

6. Cucchiara B, Tanne D, Levine SR, et al. A risk score to predict intracranial hemorrhage after recombinant tissue plasminogen activator for acute ischemic stroke. J Stroke Cerebrovasc Dis. 2008;17 (6):331-333. doi:10.1016/j.jstrokecerebrovasdis.2008.03.012

7. Lou M, Safdar A, Mehdiratta M, et al. The HAT Score: a simple grading scale for predicting hemorrhage after thrombolysis. Neurology. 2008;71(18):1417-1423. doi:10.1212/01. wn1.0000330297.58334.dd 
8. Strbian D, Engelter S, Michel P, et al. Symptomatic intracranial hemorrhage after stroke thrombolysis: the SEDAN score. Ann Neurol. 2012;71(5):634-641. doi:10.1002/ana.23546

9. Mazya M, Egido JA, Ford GA, et al. Predicting the risk of symptomatic intracerebral hemorrhage in ischemic stroke treated with intravenous alteplase: safe implementation of treatments in stroke (SITS) symptomatic intracerebral hemorrhage risk score. Stroke. 2012;43(6):1524-1531. doi:10.1161/STROKEAHA. 111.644815

10. Flint AC, Faigeles BS, Cullen SP, et al. THRIVE score predicts ischemic stroke outcomes and thrombolytic hemorrhage risk in VISTA. Stroke. 2013;44(12):3365-3369. doi:10.1161/ STROKEAHA.113.002794

11. Saposnik G, Guzik AK, Reeves M, et al. Stroke prognostication using age and NIH stroke scale: SPAN-100. Neurology. 2013;80(1):21-28. doi:10.1212/WNL.0b013e31827b1ace

12. Shariat SF, Capitanio U, Jeldres C, et al. Can nomograms be superior to other prediction tools? BJU Int. 2009;103(4):492-495. doi:10.1111/j.1464-410X.2008.08073.x

13. Zhang X, Yuan K, Wang H, et al. Nomogram to predict mortality of endovascular thrombectomy for ischemic stroke despite successful recanalization. J Am Heart Assoc. 2020;9(3):e014899. doi:10.1161/ JAHA.119.014899

14. Jehi L, Yardi R, Chagin K, et al. Development and validation of nomograms to provide individualised predictions of seizure outcomes after epilepsy surgery: a retrospective analysis. Lancet Neurol. 2015;14(3):283-290. doi:10.1016/S1474-4422(14)70325-4

15. Cappellari M, Mangiafico S, Saia V, et al. IER-SICH nomogram to predict symptomatic intracerebral hemorrhage after thrombectomy for stroke. Stroke. 2019;50(4):909-916. doi:10.1161/ STROKEAHA.118.023316

16. Cappellari M, Turcato G, Forlivesi S, et al. STARTING-SICH nomogram to predict symptomatic intracerebral hemorrhage after intravenous thrombolysis for stroke. Stroke. 2018;49(2):397-404. doi:10.1161/STROKEAHA.117.018427

17. Hacke W, Kaste M, Fieschi C, et al. Randomised double-blind placebo-controlled trial of thrombolytic therapy with intravenous alteplase in acute ischaemic stroke (ECASS II). Lancet. 1998;352 (9136):1245-1251.

18. Gumbinger C, Gruschka P, Böttinger M, et al. Improved prediction of poor outcome after thrombolysis using conservative definitions of symptomatic hemorrhage. Stroke. 2012;43(1):240-242. doi:10.1161/ STROKEAHA.111.623033
19. Hu Y, Ji C. Efficacy and safety of thrombolysis for acute ischemic stroke with atrial fibrillation: a meta-analysis. BMC Neurol. 2021;21 (1):66. doi:10.1186/s12883-021-02095-x

20. Kimura K, Iguchi Y, Shibazaki K, et al. IV t-PA therapy in acute stroke patients with atrial fibrillation. J Neurol Sci. 2009;276(12):6-8. doi:10.1016/j.jns.2008.10.018

21. Tu HT, Campbell BC, Christensen S, et al. Worse stroke outcome in atrial fibrillation is explained by more severe hypoperfusion, infarct growth, and hemorrhagic transformation. Int $J$ Stroke. 2015;10 (4):534-540. doi:10.1111/ijs.12007

22. Won SJ, Tang XN, Suh SW, et al. Hyperglycemia promotes tissue plasminogen activator-induced hemorrhage by Increasing superoxide production. Ann Neurol. 2011;70(4):583-590. doi:10.1002/ana.22538

23. Emberson J, Lees KR, Lyden P, et al. Effect of treatment delay, age, and stroke severity on the effects of intravenous thrombolysis with alteplase for acute ischaemic stroke: a meta-analysis of individual patient data from randomised trials. Lancet. 2014;384 (9958):1929-1935. doi:10.1016/S0140-6736(14)60584-5

24. Liu M, Pan Y, Zhou L, et al. Predictors of post-thrombolysis symptomatic intracranial hemorrhage in Chinese patients with acute ischemic stroke. PLoS One. 2017;12(9):e0184646. doi:10.1371/journal.pone. 0184646

25. Jickling GC, Liu D, Stamova B, et al. Hemorrhagic transformation after ischemic stroke in animals and humans. J Cereb Blood Flow Metab. 2014;34(2):185-199. doi:10.1038/jcbfm.2013.203

26. Montaner J, Molina CA, Monasterio J, et al. Matrix metalloproteinase-9 pretreatment level predicts intracranial hemorrhagic complications after thrombolysis in human stroke. Circulation. 2003;107(4):598-603. doi:10.1161/01. CIR.0000046451.38849.90

27. Maestrini I, Strbian D, Gautier S, et al. Higher neutrophil counts before thrombolysis for cerebral ischemia predict worse outcomes. Neurology. 2015;85(16):1408-1416. doi:10.1212/ WNL.0000000000002029

28. Guo Z, Yu S, Xiao L, et al. Dynamic change of neutrophil to lymphocyte ratio and hemorrhagic transformation after thrombolysis in stroke. J Neuroinflammation. 2016;13(1):199. doi:10.1186/s12974016-0680-x
Neuropsychiatric Disease and Treatment

\section{Publish your work in this journal}

Neuropsychiatric Disease and Treatment is an international, peerreviewed journal of clinical therapeutics and pharmacology focusing on concise rapid reporting of clinical or pre-clinical studies on a range of neuropsychiatric and neurological disorders. This journal is indexed on PubMed Central, the 'PsycINFO' database and CAS, and is the official journal of The International Neuropsychiatric Association (INA). The manuscript management system is completely online and includes a very quick and fair peer-review system, which is all easy to use. Visit http://www.dovepress.com/testimonials.php to read real quotes from published authors. 